\title{
Coherent transport through a resonant level coupled to random-matrix leads
}

\author{
Xinxin Yang $^{1}$ and Pei Wang $1, *$ \\ ${ }^{1}$ Department of Physics, Zhejiang Normal University, Jinhua 321004, People's Republic of China
}

(Dated: March 30, 2020)

\begin{abstract}
We study the transport through a resonant level coupled to two leads with the latter being described by Wigner's random matrices. By taking appropriate thermodynamic limit before taking the long time limit, we obtain the stationary current as a function of voltage bias. The I-V curve is similar to that of single impurity Anderson model. On the other hand, the current matrix and initial density matrix in our model look like random matrices in the eigenbasis of Hamiltonian. They satisfy the description of eigenstate thermalization hypothesis (ETH) and nonequilibrium steady state hypothesis (NESSH), respectively. A statistical formula of current has been derived based on ETH and NESSH (J. Stat. Mech.: Theo. Exp., 093105 (2017)). We check this formula in our model and find it to predict the stationary current to a high precision. The shape of I-V curve is explained by the peak structure in the characteristic function of NESSH, which is reminiscent of the transmission coefficient.
\end{abstract}

\section{INTRODUCTION}

According to statistical mechanics, the property of an equilibrium system is given by averaging over an ensemble of microscopic states. But during a measurement in laboratory, the microscopic state of system is deterministic at every moment, whose evolution follows the classical or quantum laws. Why the ensemble theory correctly predicts the measurement results has been a controversial problem since the foundation of statistical mechanics. It becomes even more sophiscated under the framework of quantum mechanics, where ergodicity breaks down due to the linearity of Schrödinger equation. A breakthrough was made by Wigner ${ }^{1}$ who proposed a random-matrix model to explain the level statistics of heavy nuclei. Following his approach, quantum chaos theory was developed $^{2-4}$. Later on, the eigenstate thermalization hypothesis (ETH) was proposed ${ }^{5,6}$ to explain the relation between ensemble theory and microscopic dynamics. ETH assumes that a laboratory system can be initialized in an arbitrary generic quantum state. But the off-diagonal elements of the initial density matrix stop contributing to the expectation of observable after a short relaxation time ${ }^{7}$. Once if the energy fluctuation is subextensive, the distribution of diagonal elements is also unimportant, because the expectation of observable with respect to eigenstates is a smooth function of energy. For an arbitrary initial state, the expectation of observable quickly relaxes to its average over a diagonal ensemble, and the latter is indeed equal to the average over a microcanonical ensemble $e^{8}$. It is the observable that thermalizes, instead of the density matrix.

During the 1980s, the development of nanotechnology pushes forward the studies on transport through mesoscopic structures ${ }^{9}-11$. The movement of electrons in these devices is basically quantum. Within the singleparticle picture, Landauer ${ }^{12,13}$ obtained a conductance formula, and Büttiker ${ }^{14}$ generalized it into the situation of multiple terminals. At the beginning of $1990 \mathrm{~s}$, the Keldysh Green's functions were widely employed to compute the conductance $\frac{15,16}{}$. Motivated by the ac- tual needs for non-perturbative calculation of conductance, researchers made efforts to derive an ensemble description for the current-carrying nonequilibrium steady states (NESS) just like the Gibbs ensemble for equilibrium states. Hershfield ${ }^{17}$ obtained a density matrix of NESS by starting from a Gibbs ensemble and then solving the Schrödinger equation in the long-time limit. The density matrix can also be obtained by maximizing the entropy subject to appropriate constraints 18 . It was later proved by Ness 19 that these approaches all result in a McLennan-Zubarev 20,21 nonequilibrium ensemble, i.e. a generalized Gibbs ensemble.

Starting from some initial state, a system will thermalize or evolve into NESS after long enough time ${ }^{22}$. Here we consider closed systems and treat leads as parts of the system when discussing mesoscopic transport. For a closed system, the quantum state remains pure for all the time. ETH explains why the expectation of observable can be predicted by an equilibrium ensemble after the system thermalizes. Similarly, one may ask why the expectation of observable (such as current) is predicted by the nonequilibrium ensemble after the system evolves into NESS. Recall that the nonequilibrium ensemble is obtained by a real-time evolution from some initial equilibrium ensemble. If we follow the logic of ETH and assume that the laboratory system can be initialized in an arbitrary generic state, the above question becomes why generic states at the initial time give the same current as an equilibrium ensemble after infinitely long evolution. In other words, why is the memory of initial state lost in the evolution to NESS?

Let us make it clear that this question cannot be answered in the same way of explaining thermalization. There is a big difference between NESS and thermalized state. For the latter, one can assume that the expectation of observable is equal to its average over the diagonal ensemble, i.e. an ensemble of eigenstates. But for NESS, this assumption must be abandoned because it rules out the possibility of nonzero current (the expectation of current with respect to diagonal ensemble is zero). The diagonal ensemble is reached only if the 
system is finite in size with nonzero level spacing, which causes the off-diagonal elements of initial density matrix being averaged out. But a finite-size system never evolves into NESS, because the initial imbalance will be removed within a finite period. The knowledge about off-diagonal elements of initial density matrix is necessary for predicting the current in NESS.

In an attempt to answer the above question, an assumption is proposed for the density matrix of a generic state, which is dubbed the nonequilibrium steady state hypothesis $(\mathrm{NESSH})^{23}$. It states that, in the eigenbasis of Hamiltonian, the off-diagonal elements of density matrix after coarse-graining become a smooth function of energies. By combining the assumption for the density matrix and ETH for the observable matrix, one derives a formula for the expectation of observable in NESS, especially the current. This formula clarifies that the observable depends only upon a few statistical features of the initial density matrix but being independent of its detail, and then makes progress in explaining why different initial states predict the same value of observable in NESS.

NESSH was verified numerically in a few models 24 , but the current formula based on it has not been checked. To check the current formula, we need a model in which ETH and NESSH stand, at the same time, the steady current can be computed precisely. For the latter purpose, the size of the system needs to be large enough and the Schrödinger or Heisenberg equation must be solved until a time that is much larger than the current relaxation time. These conditions are fulfilled in some integrable models, e.g. the single-impurity Anderson model (SIAM) without electron-electron interaction. However, to the best of our knowledge, in such models that has been studied so far, neither ETH nor NESSH stand. ETH usually stands in chaotic models in which the long-time evolution and the large system's size are not accessible simultaneously.

To circumvent this problem, we propose a model similar to SIAM but with two leads being replaced by random matrices. After the replacement, the single-particle density matrix satisfies NESSH, while the current matrix in the single-particle eigenbasis satisfies ETH. It is worth mentioning that, the application of random matrices in modeling the mesoscopic transport has a long history, which was originally motivated by the need to explain universal conductance fluctuations ${ }^{25}$ and becomes a regular tool today ${ }^{26-28}$. But in previous studies, it is the scattering region which is described by a random matrix, instead of the leads. In these studies, the whole system does not display features of ETH or NESSH, because the leads are overwhelmingly large compared to the scattering region. For ETH and NESSH to stand, we model the leads by random matrices. This can also be justified from the aspect of experiments. The leads manufactured in mesoscopic experiments indeed have irregular shapes, unavoidable impurities, electron-electron interaction and electron-phonon interaction. It is then reasonable to ex- pect that the level statistics of leads is similar to that of the Wigner's random matrix.

The rest of paper is organized as follows. We review the assumptions and formula of ETH and NESSH in Sec. II] Especially, we explain how to derive the current formula. Our model of random-matrix leads is introduced in Sec. III together with the current operator. In Sec. IV, it is made clear that both ETH and NESSH stand in our model, and we explain how to obtain the characteristic functions which are needed in the current formula. In Sec. $\mathrm{V}$, the current is obtained by using the current formula. A comparison with the ab-initio calculation is presented to check the current formula. Sec. VI is a summary.

\section{ETH, NESSH AND CURRENT FORMULA}

In this section, we shortly review the formula of ETH and NESSH. Our review is based on the references 7] and 23].

Let us consider a system which is prepared in a generic quantum state denoted by $\left|\Psi_{0}\right\rangle$. The density matrix is correspondingly $\hat{\rho}_{0}=\left|\Psi_{0}\right\rangle\left\langle\Psi_{0}\right|$. Here a few words are needed for explaining which states are "generic". Obviously, if we consider the equal-weight superposition of two eigenstates with different energies, it neither thermalizes nor evolves into NESS. But such a well-tuned many-body state is hard if not impossible to realize in experiments. It is not generic. Due to the unavoidable interaction between particles in a laboratory system, the eigenstate usually involves huge number of natural bases. And generally speaking, the prepared initial state is either an eigenstate (such as the ground state) or a superposition of huge number of eigenstates. The latter is called "generic" in this paper.

We use $\hat{H}$ to denote the Hamiltonian and the Greek letters such as $\alpha$ and $\beta$ to denote the eigenstates with the eigenenergies $E_{\alpha}$ and $E_{\beta}$, respectively. The evolution of density matrix follows the quantum Liouville equation, reading

$$
\hat{\rho}(t)=e^{-i \hat{H} t} \hat{\rho}_{0} e^{i \hat{H} t} .
$$

Suppose that $\hat{I}$ is an observable operator. Its expectation value at time $t$ is given by $I(t)=\operatorname{Tr}[\hat{\rho}(t) \hat{I}]$.

One is interested in the fate of $I(t)$ in the asymptotically long time. An argument based on ETH shows that $I(t)$ must relax to a stationary value which is defined as $I=\lim _{t \rightarrow \infty} I(t)$. And this stationary value is equal to the long-time average of $I(t)$, which can be expressed as

$$
\begin{aligned}
I & =\lim _{T \rightarrow \infty} \frac{1}{T} \int_{0}^{T} d t e^{i t\left(E_{\alpha}-E_{\beta}\right)} \sum_{\alpha, \beta}\left[\left\langle\beta\left|\hat{\rho}_{0}\right| \alpha\right\rangle\langle\alpha|\hat{I}| \beta\rangle\right] \\
& =\sum_{\alpha}\left[\left\langle\alpha\left|\hat{\rho}_{0}\right| \alpha\right\rangle\langle\alpha|\hat{I}| \alpha\rangle\right] .
\end{aligned}
$$


Eq. (2) shows that the off-diagonal elements - $\left\langle\beta\left|\hat{\rho}_{0}\right| \alpha\right\rangle$ with $\alpha \neq \beta$ do not contribute to $I$. For obtaining this conclusion, we have to assume $E_{\alpha} \neq E_{\beta}$ for arbitrary $\alpha \neq \beta$ and $\left|E_{\alpha}-E_{\beta}\right|$ has a nonzero lower bound. This nondegeneracy condition is fulfilled in a generic system if the system's size is finite. Usually, the symmetries must be broken for nondegeneracy, which is considered as a prerequisite in the discussion of thermalization.

ETH assumes that the matrix of $\hat{I}$ in the eigenbasis of $\hat{H}$ can be written as

$$
\begin{aligned}
I_{\alpha, \beta} & =\langle\alpha|\hat{I}| \beta\rangle \\
& =I(\bar{E}) \delta_{\alpha, \beta}+D^{-1 / 2}(\bar{E}) f_{I}(\bar{E}, \omega) R_{\alpha \beta}^{I},
\end{aligned}
$$

where $\bar{E}=\left(E_{\alpha}+E_{\beta}\right) / 2$ is the average energy, $\omega=$ $E_{\alpha}-E_{\beta}$ is the energy difference, and $D(\bar{E})$ is the density of eigenstates. $I(\bar{E})$ and $f_{I}(\bar{E}, \omega)$ are smooth functions. $R_{\alpha \beta}^{I}$ is a random number with zero mean and unit variance. The first and second terms of Eq. (3) are for the diagonal and off-diagonal elements, respectively. Crucially, Eq. (3) tells us that the diagonal elements are a smooth function of $\bar{E}$. It is natural to assume that the energy fluctuation of the initial state is subextensive, hence, $\rho_{\alpha, \alpha}=\left\langle\alpha\left|\hat{\rho}_{0}\right| \alpha\right\rangle$ is significant only within a small energy shell centered at $E\left(\hat{\rho}_{0}\right)=\operatorname{Tr}\left[\hat{\rho}_{0} \hat{H}\right]$, i.e. the energy of initial state. Therefore, the sum of $\alpha$ in Eq. (2) can be treated as a sum over this energy shell, within which $I_{\alpha, \alpha}$ is approximately a constant due to the smoothness of $I(\bar{E})$. By using the relation $\sum_{\alpha} \rho_{\alpha, \alpha} \equiv 1$, one then concludes that $I$ is equal to the value of $I(\bar{E})$ at $\bar{E}=E\left(\hat{\rho}_{0}\right)$. Furthermore, since $I_{\alpha, \alpha}$ is a constant, $I$ must be also equal to the average of $I_{\alpha, \alpha}$ over this energy shell, which is just the average over the microcanonical ensemble. The expectation of observable finally relaxes to its value in a thermal ensemble.

Now we turn to NESS. A typical system hosting NESS includes two leads which are connected to each other through a central scattering regime - a model that was frequently used in the study of mesoscopic transport. We are still interested in the expectation of an observable in the long time limit. We choose an alternative strategy to evaluate $I$. We start from $I(t)$ which is

$$
I(t)=\sum_{\alpha} \rho_{\alpha, \alpha} I_{\alpha, \alpha}+\sum_{\alpha \neq \beta} e^{-i \omega t} \rho_{\alpha, \beta} I_{\beta, \alpha} .
$$

The first term of Eq. (4) comes from the diagonal elements, being equal to what we obtain from Eq. (2). From now on, we use $I_{e q}$ to denote $\sum_{\alpha} \rho_{\alpha, \alpha} I_{\alpha, \alpha}$. If the second term of Eq. (4) decays to zero in the limit $t \rightarrow \infty$, the observable thermalizes and we find $I=I_{e q}$. But if the second term relaxes to a nonzero value, the system evolves into NESS instead and we have $I \neq I_{e q}$. We use $I_{n e}(t)$ to denote the second term, Eq. (4) is then rewritten as $I(t)=I_{e q}+I_{n e}(t)$.

NESSH assumes that $\rho_{\alpha, \beta}=\left\langle\alpha\left|\hat{\rho}_{0}\right| \beta\right\rangle$ for a generic state can be expressed as

$$
\rho_{\alpha, \beta}=p(\bar{E}) \delta_{\alpha, \beta}+D^{-3 / 2}(\bar{E}) f(\bar{E}, \omega) R_{\alpha \beta},
$$

where $p(\bar{E})$ is a smooth function. $f(\bar{E}, \omega)$ is called the dynamical characteristic function, which is smooth almost everywhere except for a measure-zero set in the $\bar{E}-\omega$ plane. As similar as $R_{\alpha \beta}^{I}, R_{\alpha \beta}$ is a random number of zero mean and unit variance. Again, the first and second terms of Eq. (5) are for the diagonal and off-diagonal elements, respectively. In other words, the second term is only for $\alpha \neq \beta$. By combining the assumptions (3) and (5), we obtain an expression of $I_{n e}(t)$ which reads

$$
I_{n e}(t)=\sum_{\alpha \neq \beta} e^{-i \omega t} \frac{f(\bar{E}, \omega) f_{I}(\bar{E},-\omega)}{D^{2}(\bar{E})} R_{\alpha \beta} R_{\beta \alpha}^{I} .
$$

Next we divide the $E_{\alpha}-E_{\beta}$ plane (or $\bar{E}-\omega$ plane) into many tiny boxes, with each one still containing a great number of $\{(\alpha, \beta)\}$. The functions $f, f_{I}$ and $D$ can be treated as constants within each box, because they are smooth functions of energy and the boxes are small. On the other hand, $R_{\alpha \beta}$ and $R_{\beta \alpha}^{I}$ are random numbers depending on the microscopic states $\alpha$ and $\beta$. In each box, $R_{\alpha \beta} R_{\beta \alpha}^{I}$ distributes diversely. And we define the correlation function $\mathcal{C}$ to be

$$
\mathcal{C}=\frac{1}{M} \sum_{(\alpha, \beta) \in b o x} R_{\alpha \beta} R_{\beta \alpha}^{I},
$$

where the sum is over a box centered at $\left(E_{\alpha}, E_{\beta}\right)$ and $M$ is the total number of $(\alpha, \beta)$ within this box. It is natural to assume that $\mathcal{C}$ is a smooth function of $E_{\alpha}$ and $E_{\beta} . \mathcal{C}$ is indeed the mean of the random number $R_{\alpha \beta} R_{\beta \alpha}^{I}$.

As the system's size increases, the level spacing between neighbor eigenenergies vanishes gradually, we can then replace the sum in Eq. (6) by integral: $\sum_{\alpha} \rightarrow$ $\int d E_{\alpha} D\left(E_{\alpha}\right)$. Here we exclude the presence of isolated energy levels and assume that $D$ is a regular function of energy, which is usually true in the study of transport. Moreover, we can change variables in the integral by using $\int d E_{\alpha} d E_{\beta}=\int d \bar{E} d \omega$. $I_{n e}(t)$ becomes

$$
\begin{aligned}
I_{n e}(t)=\int d \bar{E} d \omega & e^{-i \omega t} \frac{D(\bar{E}+\omega / 2) D(\bar{E}-\omega / 2)}{D^{2}(\bar{E})} \\
& \times f(\bar{E}, \omega) f_{I}(\bar{E},-\omega) \mathcal{C}(\bar{E}, \omega) .
\end{aligned}
$$

It is prepared to discuss the long time limit of $I_{n e}(t)$, denoted by $I_{n e}=\lim _{t \rightarrow \infty} I_{n e}(t)$. The integral with respect to $\omega$ is crucial. Obviously, once if the system's size is finite, $|\omega|=\left|E_{\alpha}-E_{\beta}\right|$ has a nonzero lower bound. That is to say that the integrand in Eq. (8) vanishes for $\omega$ being close to zero. According to Riemann-Lebesgue lemma, once if the integrand has no singularity at $\omega=0$, an integral of the type $\int d \omega e^{-i \omega t}$ must vanish as $t \rightarrow \infty$. This is what we expect, since a finite system always thermalizes and $I_{n e}$ must be zero.

To see the conditions under which $I_{n e}$ is nonzero, we need to know how a NESS is realized. Let us consider a system consisting of two leads which are connected to a scattering regime. Once if the leads are finite in length, the system always thermalizes as $t \rightarrow \infty$. If the initial 
chemical potentials of two leads are different, the system will evolve into a current-carrying quasi-steady state and stays there for some time, before it finally thermalizes. If we increase the length of leads, the lifetime of the quasisteady state also increases. As the length of leads becomes infinite (thermodynamic limit), the quasi-steady state becomes a true steady state, i.e. NESS. Therefore, the thermodynamic limit must be taken before $t \rightarrow \infty$, if we hope to obtain a NESS.

In the thermodynamic limit, the level spacing vanishes. In Eq. (8), this means that the lower bound of $|\omega|=\left|E_{\alpha}-E_{\beta}\right|$ vanishes, and the integrand can be singular at $\omega=0$. The second assumption of NESSH states that, an initial state evolves into NESS if its dynamical characteristic function is singular at $\omega=0$ with the form

$$
f(\bar{E}, \omega)=\frac{\rho(\bar{E}, \omega)}{|\omega|},
$$

where $\rho(\bar{E}, \omega)$ is continuous at $\omega=0$. To make further progress, we need to discuss the symmetry of $\rho(\bar{E}, \omega)$ and $f_{I}(\bar{E}, \omega)$. For simplicity, we suppose that the Hamiltonian matrix is real, which is typical in the absence of magnetic field. The eigenvectors $|\alpha\rangle$ or $|\beta\rangle$ are now real vectors. If the initial state $\left|\Psi_{0}\right\rangle$ is a real vector, the matrix elements $\rho_{\alpha, \beta}$ are all real. And from the hermitianity of $\hat{\rho}_{0}$, we know $\rho_{\alpha, \beta}=\rho_{\beta, \alpha}$, which requires that $\rho(\bar{E}, \omega)=\rho(\bar{E},-\omega)$ be an even function of $\omega$ according to Eq. (5). Note that $R_{\alpha, \beta}=R_{\beta, \alpha}$ is real. Furthermore, we consider $\hat{I}$ to be the current operator. It is usually defined as the change rate of particle number in one lead. It is well known that such an operator is purely imaginary, i.e. $I_{\alpha, \beta}$ is a purely imaginary number. From the hermitianity of $\hat{I}$, we obtain $I_{\alpha, \beta}=I_{\beta, \alpha}^{*}=-I_{\beta, \alpha}$, which requires that $f_{I}(\bar{E}, \omega)=-f_{I}(\bar{E},-\omega)$ be purely imaginary and an odd function of $\omega$. Note that $R_{\alpha, \beta}^{I}=R_{\beta, \alpha}^{I}$ is real.

Substituting Eq. (9) into Eq. (8) and taking the limit $t \rightarrow \infty$, we immediately find

$$
I_{n e}=i \pi \int d \bar{E} \rho(\bar{E}, 0) f_{I}\left(\bar{E}, 0^{+}\right) \mathcal{C}(\bar{E}, 0),
$$

where $f_{I}\left(\bar{E}, 0^{+}\right)=\lim _{\omega \rightarrow 0^{+}} f_{I}(\bar{E}, \omega)$. To obtain Eq. (10), we change the variable $\omega t \rightarrow x$ and use the relation $\int_{-\infty}^{\infty} d x e^{i x} / x=i \pi$. The functions $\rho$ and $\mathcal{C}$ are continuous at $\omega=0$, but $f_{I}(\bar{E}, \omega)$ is not. This is why $f_{I}\left(\bar{E}, 0^{+}\right)$ appears in Eq. (10). At the same time, $I_{e q}$ for the current operator must be zero. To evaluate $I_{e q}$, we start from a finite system in which the eigenstates and eigenenergies are well-defined, and then take the thermodynamic limit. But in a finite system, the expectation of current operator with respect to eigenstates is zero, i.e., the diagonal elements $I_{\alpha, \alpha}$ are all zero. We then obtain $I_{e q}=0$ for arbitrary system's size, thereafter, its thermodynamic limit must also be zero. The stationary current then becomes

$$
I=I_{n e}
$$

Eq. (10) is the current formula of NESSH. It tells us that most elements of initial density matrix do not contribute to the current in NESS. First, $I$ is independent of the diagonal elements. Second, among the off-diagonal elements, those with finite $\left|E_{\alpha}-E_{\beta}\right|$ have no contribution, since both $\rho(\bar{E}, 0)$ and $\mathcal{C}(\bar{E}, 0)$ depend only upon $\rho_{\alpha, \beta}$ in the asymptotic limit $\left|E_{\alpha}-E_{\beta}\right| \rightarrow 0$. And $I$ is indirectly connected to $\rho_{\alpha, \beta}$ with infinitesimal energy difference. $\rho(\bar{E}, 0)$ is obtained by averaging $\rho_{\alpha, \beta}$ over a small energy box, being then independent of the distribution of $\rho_{\alpha, \beta}$ within the box. And $\mathcal{C}(\bar{E}, 0)$ is the correlation between $\rho_{\alpha, \beta}$ and $I_{\alpha, \beta}$ within the box. Eq. (10) shows that the current in NESS is determined by a few statistics of initial density matrix and current matrix. This result is reminiscent of a thermalization process, during which the memory of initial microscopic state is lost and finally the system's properties are determined by a few macroscopic parameters. But NESS must be distinguished from an equilibrium state. A finite stationary current survives in NESS but not in thermal equilibrium.

\section{MODEL}

We propose a model to check the current formula (10). Our purpose is to compare the stationary current obtained from Eq. (10) with that obtained from an ab-initio calculation. The prerequisite of using Eq. (10) is that ETH and NESSH stand. On the other hand, an abinitio calculation requires us to solve the Schrödinger or Heisenberg equation for $I(t)$. The thermodynamic limit and $t \rightarrow \infty$ are then taken in turn. In the ab-initio calculation, we need to know $I(t)$ at both large $t$ and large system's size. In our model, the prerequisite of Eq. (10) is fulfilled and an ab-initio calculation is accessible.

Our model is composed of a resonant level and two leads. Crucially, the Hamiltonians of the leads are random matrices. We start from two independent random matrices $A_{1}$ and $A_{2}$, which are for the left and right leads, respectively. The distribution of $A_{i}(i=1,2)$ is that of a Gaussian orthogonal ensemble (GOE) ${ }^{29}$ with the probability density $P\left(A_{i}\right) \propto \exp \left[-\operatorname{Tr}\left(A_{i}^{2}\right) / 2 \sigma^{2}\right]$, where $\sigma$ is related to the averaged level spacing in leads. We diagonalize $A_{i}$ and obtain a series of eigenvalues $\epsilon_{i k}$. The Hamiltonians of leads are then expressed in the eigenbasis as

$$
\hat{H}_{i}=\sum_{k} \epsilon_{i k} \hat{c}_{i k}^{\dagger} \hat{c}_{i k}
$$

where $\epsilon_{i k}$ denotes the energy levels of leads and $\hat{c}_{i k}$ and $\hat{c}_{i k}^{\dagger}$ are the fermionic field operators. $\epsilon_{i k}$ satisfies the wellknown Wigner-Dyson distribution with the probability density $^{29}$

$$
P\left(\epsilon_{i 1}, \epsilon_{i 2}, \cdots, \epsilon_{i N_{0}}\right) \propto e^{-\frac{\epsilon_{i 1}^{2}+\cdots+\epsilon_{i N_{0}}^{2}}{2 \sigma^{2}}}\left|\prod_{k^{\prime}>k}\left(\epsilon_{i k^{\prime}}-\epsilon_{i k}\right)\right| .
$$


$N_{0}$ denotes the dimension of $A_{i}$, which can be treated as the lead's size. The thermodynamic limit is defined to be $N_{0} \rightarrow \infty$. The averaged level spacing in leads is proportional to $\sigma$ according to random matrix theory. The proper way of taking thermodynamic limit is to increase the lead's size while keeping its bandwidth invariant. Therefore, we keep $N_{0} \sigma$ a constant as $N_{0}$ increases. In the numerical simulation, large level spacing appears at the spectrum edge of $A_{i}$, which causes the density of states ill-defined. To circumvent this problem, we keep only $\epsilon_{i k}$ that lies within an interval $[-\Delta, \Delta]$ where $2 \Delta$ denotes the lead's bandwidth. $\Delta$ should be small compared to the largest eigenvalue of $A_{i}$, so that $\epsilon_{i k}$ distributes densely within the interval $[-\Delta, \Delta]$.

The Hamiltonian of the resonant level is $\hat{H}_{d}=\epsilon_{d} \hat{d}^{\dagger} \hat{d}$. The coupling between the leads and the resonant level is set to a random number. The corresponding Hamiltonian is $\hat{H}_{c}=\sum_{i, k} g_{i k}\left(\hat{c}_{i k}^{\dagger} \hat{d}+H . c.\right)$. Here $g_{i k}$ is an independent random number which has a Gaussian distribution with the probability density $P\left(g_{i k}\right)=$ $\exp \left(-\left(g_{i k}\right)^{2} / 2 \sigma_{t}^{2}\right) / \sqrt{2 \pi \sigma_{t}^{2}}$, where $\sigma_{t}$ denotes the coupling strength. The total Hamiltonian is written as

$$
\hat{H}=\hat{H}_{1}+\hat{H}_{2}+\hat{H}_{d}+\hat{H}_{c} \text {. }
$$

We set $\epsilon_{j k}$ and $g_{i k}$ to be independent random numbers for ETH and NESSH to hold. Otherwise, if the system has an explicit parity symmetry, i.e. $g_{1 k}=g_{2 k}$ and $\epsilon_{1 k}=\epsilon_{2 k}, \hat{H}$ can be decoupled by defining the (anti)symmetric basis $\hat{c}_{k \pm}=\left(\hat{c}_{1 k} \pm \hat{c}_{2 k}\right) / \sqrt{2}$, and then ETH and NESSH break down. By using random levels and random couplings, we keep the parity symmetry in a statistical sense. Furthermore, for a resonant level coupled to leads, one usually defines the broadening of resonant level as $\Gamma(E)=\sum_{i k} \pi \delta\left(E-\epsilon_{i k}\right) g_{i k}^{2}$. The mean of $g_{i k}^{2}$ is defined to be $\sigma_{t}^{2}$. It is then straight forward to obtain $\Gamma=2 \pi D_{l} \sigma_{t}^{2}$, where $D_{l}$ is the density of states in one lead. To take the proper thermodynamic limit, we must keep $\Gamma$ invariant as the lead's size increases. Therefore, $\sqrt{D_{l}} \sigma_{t}$ must be a constant as $N_{0}$ increases.

Our model excludes interaction between particles, otherwise, an ab-initio calculation is hard to carry out. In the absence of interaction, ETH and NESSH do not stand for the many-body eigenstates. But they do stand under the single-particle picture. If we use $\hat{\rho}$ to denote the density matrix of a single particle and $\alpha$ and $\beta$ to denote the single-particle eigenstates, $\rho_{\alpha, \beta}$ and $I_{\alpha, \beta}$ satisfy Eq. (5) and Eq. (3), respectively. At the same time, due to the lack of interaction, the ab-initio calculation of current is easy once if we suppose the initial state to be a product of single-particle states.

In a two-lead model, the current operator $\hat{I}$ is usually defined as the changing rate of particle number, reading

$$
\begin{aligned}
\hat{I} & =\frac{1}{2}\left(\frac{d \hat{N}_{2}}{d t}-\frac{d \hat{N}_{1}}{d t}\right) \\
& =\frac{i}{2} \sum_{j k}(-1)^{j} g_{j k}\left(\hat{c}_{j k}^{\dagger} \hat{d}-\hat{d}^{\dagger} \hat{c}_{j k}\right),
\end{aligned}
$$

where $\hat{N}_{j}=\sum_{k} \hat{c}_{j k}^{\dagger} \hat{c}_{j k}$ is the number of particles in lead $j$, and $i$ is the imaginary unit. For obtaining a currentcarrying NESS, we employ next initial state. At time $t=0$, the levels between $-V / 2$ and $V / 2$ in lead 1 are occupied, while all the other levels of lead 1 or lead 2 are empty. The initial state is a product of left-lead occupied levels. If we are only interested in the stationary current but not how $I(t)$ relaxes, such defined initial state is equivalent to the initial condition under which the left and right leads are at zero temperature with Fermi energies $V / 2$ and $-V / 2$, respectively. The latter condition is what one usually adopts in the study of mesoscopic transport. $V$ has the meaning of voltage bias. The equivalence between two initial conditions is due to the fact that the current contributed by the levels lower than $-V / 2$ in lead 1 neutralizes the current contributed by the occupied levels in lead 2 as $t \rightarrow \infty$.

We use $\hat{\rho}^{j k}$ to denote the density matrix of a particle occupying the level $\epsilon_{j k}$ in lead $j$. In the absence of particle-particle interaction, the total current can be expressed as

$$
I(t)=\sum_{j k} I^{j k}(t)
$$

where $I^{j k}(t)=\operatorname{Tr}\left[\hat{I} \hat{\rho}^{j k}(t)\right]$ is the current contributed by a single particle and the sum is over all the occupied levels at $t=0$. By inserting the single-particle eigenstates $\alpha$ and $\beta$ into $\operatorname{Tr}\left[\hat{I} \hat{\rho}^{j k}(t)\right]$, Eq. (16) can be reexpressed as

$$
I(t)=\sum_{\alpha \neq \beta} e^{-i\left(E_{\alpha}-E_{\beta}\right) t} \rho_{\alpha, \beta} I_{\beta, \alpha}
$$

where $\rho_{\alpha, \beta}=\sum_{j, k} \rho_{\alpha, \beta}^{j k}$. And $\rho_{\alpha, \beta}^{j k}=\left\langle\alpha\left|\hat{\rho}^{j k}\right| \beta\right\rangle$ and $I_{\beta, \alpha}=\langle\beta|\hat{I}| \alpha\rangle$ are the single-particle density matrix and current matrix, respectively. According to the definition of current operator, it is easy to see $I_{\alpha \alpha}=0$. This explains why the terms with $\alpha=\beta$ are excluded in Eq. (17). Eq. (17) has the same form as Eq. (4), except that $\rho_{\alpha, \beta}$ in Eq. (17) is a sum of single-particle density matrices instead of a many-body density matrix. We find that such defined $\rho_{\alpha, \beta}$ satisfies Eq. (5), therefore, the current formula (10) should stand in our model. To check the current formula is to compare Eq. (10) with the current obtained from Eq. (17) by taking $t \rightarrow \infty$ after $N_{0} \rightarrow \infty$.

It is worth mentioning that sampling $g_{j k}$ or $A_{j}$ for multiple times is unnecessary even they are random numbers or matrices. Once if $N_{0}$ is large enough, NESSH and ETH stand in each shot of sampling, and the current formula 


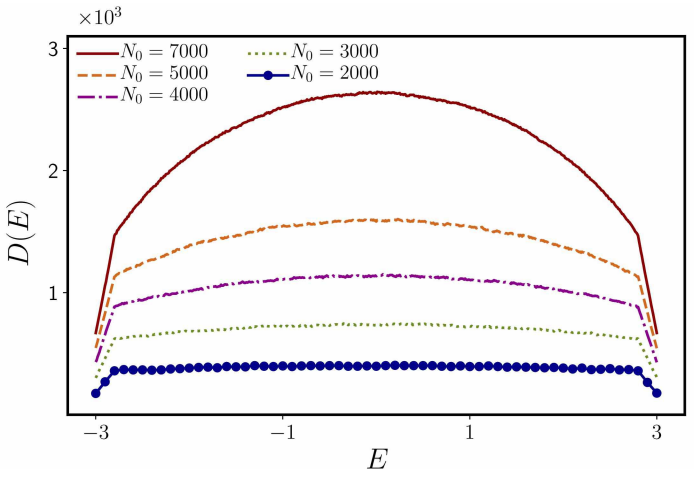

FIG. 1. (Color online) The density of states for different $N_{0}$. We set $\epsilon_{d}=0, \Delta=3, N_{0} \sigma=200$ and $\sqrt{D_{l}} \sigma_{t}=\sqrt{2 / 5}$.

can be checked by a single sampling. Moreover, sampling for multiple times has little effect on the results of current. We then do not carry out the sampling average in this paper.

\section{EVIDENCE OF ETH AND NESSH}

In this section, we show that ETH and NESSH stand in our model. And we compute the functions $D(E)$, $f_{I}(\bar{E}, \omega), f(\bar{E}, \omega)$ and $\mathcal{C}(\bar{E}, \omega)$, which are the characteristic functions of our model. These functions are necessary in the application of current formula (10).

The free parameters of our model include $N_{0}, \Delta, N_{0} \sigma$, $\sqrt{D_{l}} \sigma_{t}$ and $\epsilon_{d}$. We assign to each parameter a value. The numerical simulation starts from generating the random matrices $A_{i}$ with respect to given $N_{0}$ and $\sigma$. By diagonalizing $A_{i}$ we obtain all the levels within lead 1 and 2 . $D_{l}$ is the averaged density of states in lead 1, which is also equal to the averaged density of states in lead 2 . We first compute $D_{l}(E)$ at a specific energy $E$ by counting the number of levels within a box of length 0.4 centered at $E$. We then obtain $D_{l}$ from $D_{l}=\frac{1}{2 \Delta} \int_{-\Delta}^{\Delta} d E D_{l}(E)$. Since $\sqrt{D_{l}} \sigma_{t}$ is a predefined parameter, the value of $\sigma_{t}$ is obtained. Note that $g_{i k}$ is an independent random number with normal distribution of variance $\sigma_{t}^{2}$. We can now generate $g_{i k}$ and then the Hamiltonian matrix (14) in the single-particle basis.

Diagonalizing this matrix, we obtain all the eigenvectors and eigenenergies. The density of states $D(E)$ is obtained by choosing an energy box of length 0.4 centered at $E$ and then counting the eigenenergies falling within it. Here the size of box is chosen so that each box contains a few hundreds of levels. Fig. 1 plots $D(E)$ for different $N_{0}$. We see that $D(E)$ changes smoothly and reaches a maximum at $E=0$. And the density of states increases with the lead's size, as we expect.

After we obtain all the eigenvectors, the current matrix can be computed according to $I_{\alpha, \beta}=\langle\alpha|\hat{I}| \beta\rangle$. Notice that $I_{\alpha, \beta}$ is a purely imaginary number. If one observes
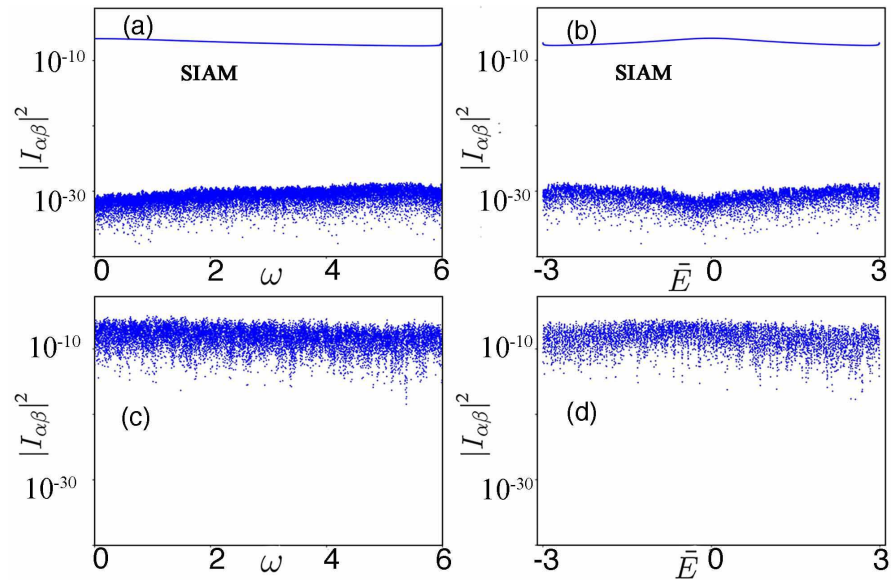

FIG. 2. (Color online) (a) $\left|I_{\alpha, \beta}\right|^{2}$ vs $\omega$ for a small window $\bar{E} \in(-0.01,0.01)$ in the SIAM. (b) $\left|I_{\alpha, \beta}\right|^{2}$ vs $\bar{E}$ for a small window $\omega \in(0,0.01)$ in the SIAM. (c) $\left|I_{\alpha, \beta}\right|^{2}$ vs $\omega$ for a small window $\bar{E} \in(-0.01,0.01)$ in our model. (d) $\left|I_{\alpha, \beta}\right|^{2}$ vs $\bar{E}$ for a small window $\omega \in(0,0.01)$ in our model. The parameters of SIAM can be found in Appendix A For our model (14), we set $\Delta=3, \epsilon_{d}=0, N_{o} \sigma=200, N_{0}=2000$ and $\sqrt{D_{l}} \sigma_{t}=1 / \sqrt{10}$.

the distribution of $I_{\alpha, \beta}$, it becomes clear that ETH stands in our model. The diagonal elements of the current matrix are all zero, indicating $I(\bar{E}) \equiv 0$ in Eq. (3). While Fig. 2 plots the off-diagonal elements. To be specific, Fig. 2 plots $\left|I_{\alpha, \beta}\right|^{2}$ vs $\omega=E_{\alpha}-E_{\beta}$ for a small window $\bar{E}=\left(E_{\alpha}+E_{\beta}\right) / 2 \in(-0.01,0.01)$, and also $\left|I_{\alpha, \beta}\right|^{2}$ vs $\bar{E}$ for a window $\omega \in(0,0.01)$. As a comparison, $\left|I_{\alpha, \beta}\right|^{2}$ in the SIAM is plotted in the same figure. The definition of SIAM can be found in Appendix A. The off-diagonal elements of current matrix are qualitatively different in SIAM and our model. In the SIAM, a large fraction of off-diagonal elements vanish $\left(\left|I_{\alpha, \beta}\right|^{2} \sim 10^{-30}\right)$, at the same time, a small fraction of elements are significant $\left(\left|I_{\alpha, \beta}\right|^{2} \sim 10^{-10}\right)$. Conversely, we do not find large outliers in the off-diagonal elements of our model.

If Eq. (3) stands, one must have $\left|I_{\alpha, \beta}\right|^{2}=$ $D^{-1}\left|f_{I}\right|^{2}\left|R_{\alpha \beta}^{I}\right|^{2}$, where $D$ and $f_{I}$ are smooth functions of $\bar{E}$ and $\omega$ and $\left|R_{\alpha \beta}^{I}\right|^{2}$ is a random number of unit mean. As a consequence, $\left|I_{\alpha, \beta}\right|^{2}$ should distribute densely around a smooth curve, i.e. $D^{-1}\left|f_{I}\right|^{2}$. Obviously, $I_{\alpha, \beta}$ in SIAM cannot be described by Eq. (3). This is a result of SIAM's integrability. $I_{\alpha, \beta}$ in SIAM is significant only for few $(\alpha, \beta)$ which are connected to the symmetry of the model. On the other hand, these symmetries are broken in our model, thereafter, $I_{\alpha, \beta}$ in our model can be described by Eq. (3), as clearly shown in Fig. 2].

The function $f_{I}(\bar{E}, \omega)$ is obtained by averaging $\left|I_{\alpha, \beta}\right|^{2}$ over an energy box. It is straight forward to see

$$
\left|f_{I}(\bar{E}, \omega)\right|^{2}=\overline{\left|I_{\alpha \beta}\right|^{2}} D(\bar{E})
$$


where $\overline{\left|I_{\alpha \beta}\right|^{2}}$ denotes the average over a square box centered at $(\bar{E}, \omega)$ with the edge chosen to 0.2 . Such a box usually contains thousands of $(\alpha, \beta)$. Eq. (18) does not tell us the sign of $f_{I}$, which can be decided freely. In the derivation of Eq. (10), we have shown that $f_{I}$ is purely imaginary and an odd function of $\omega$. Once if these properties are guaranteed, the sign of $f_{I}$ is unimportant, because it can always be absorbed into $R_{\alpha, \beta}^{I}$.
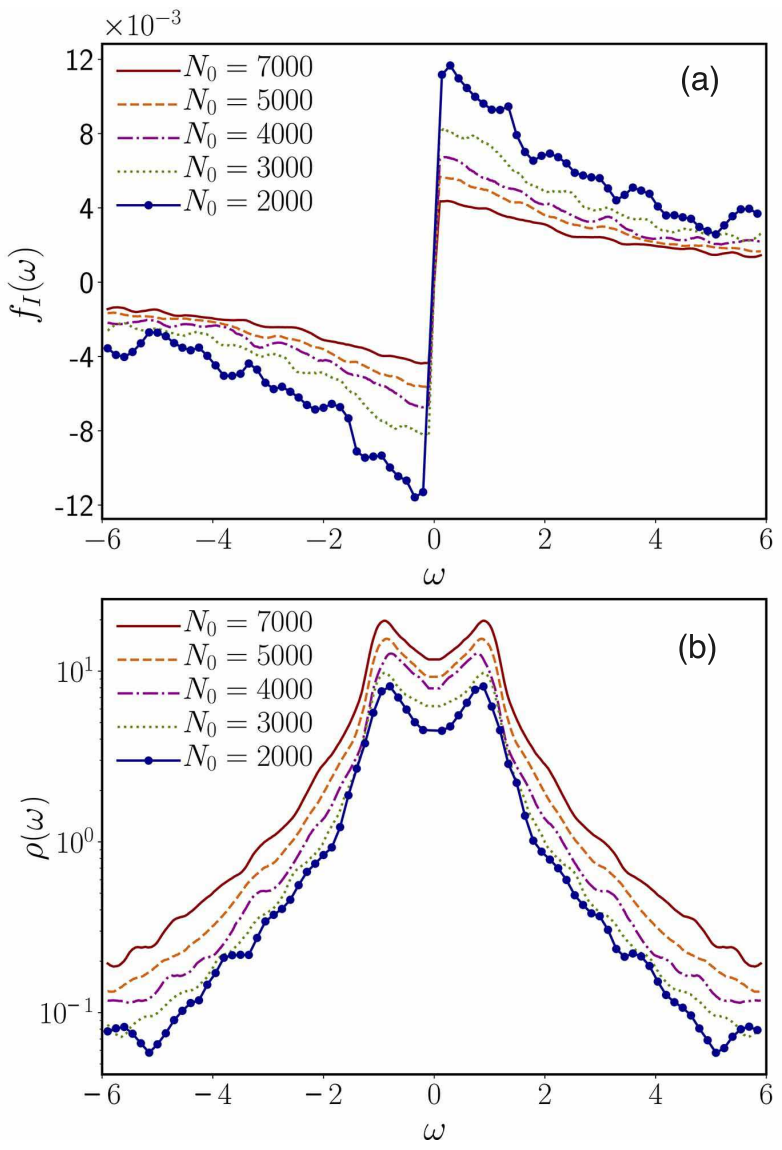

FIG. 3. (Color online) (a) The function $f_{I}(0, \omega)$ for different $N_{0}$. (b) The function $\rho(0, \omega)$ for different $N_{0}$. The parameters are chosen to $\Delta=3, \epsilon_{d}=0, N_{0} \sigma=200, V=1$ and $\sqrt{D_{l}} \sigma_{t}=$ $1 / \sqrt{10}$, which corresponds to $\Gamma=0.2 \pi$.

Fig. 3 the top panel plots the imaginary part of $f_{I}$ as a function of $\omega$ with $\bar{E}=0$ fixed. For $\omega>0, f_{I}$ changes smoothly. And the change is smoothened as $N_{0}$ increases. In the limit $\omega \rightarrow 0^{+}, f_{I}$ approaches a nonzero constant, which is denoted by $f_{I}\left(\bar{E}, 0^{+}\right)$. It is not difficult to determine $f_{I}\left(\bar{E}, 0^{+}\right)$numerically, because $f_{I}$ changes slowly as $\omega \rightarrow 0^{+}$(see the curve at $N_{0}=7000$ ). Moreover, $f_{I}(\omega)$ is an odd function, hence, it must be discontinuous at $\omega=0$. This explains why we use $f_{I}\left(\bar{E}, 0^{+}\right)$instead of $f_{I}(\bar{E}, 0)$, because the latter is not well-defined. The discontinuity at $\omega=0$ does not cause trouble in determining $f_{I}\left(\bar{E}, 0^{+}\right)$. Because what we indeed calculate is $\left|f_{I}\right|^{2}$, which is continuous at $\omega=0$.

Next we discuss the density matrix $\rho_{\alpha, \beta}=\langle\alpha|\hat{\rho}| \beta\rangle$. Note that $\rho_{\alpha, \beta}$ is real. Fig. 4 plots the off-diagonal ele-
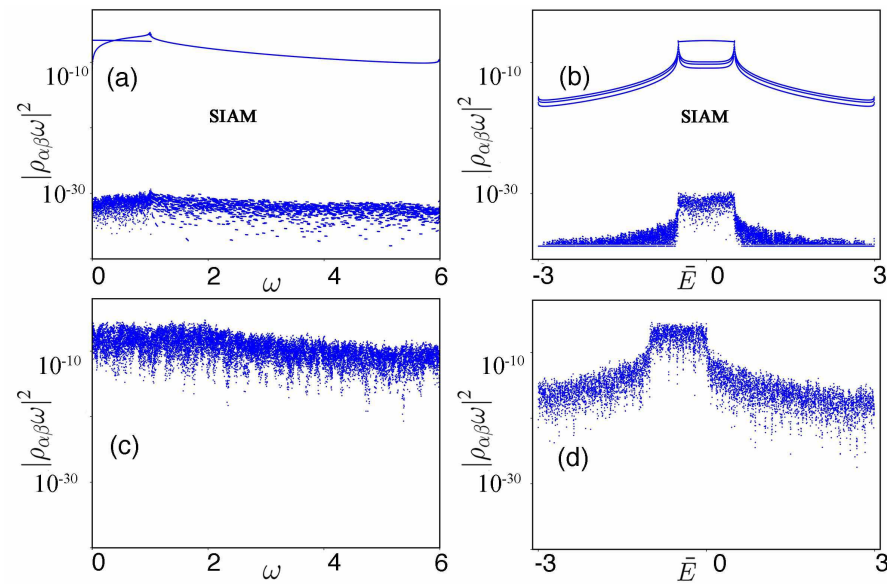

FIG. 4. (Color online) (a) $\left|\rho_{\alpha, \beta}\right|^{2} \omega^{2}$ vs $\omega$ for a window $\bar{E} \in$ $(-0.01,0.01)$ in the SIAM. (b) $\left|\rho_{\alpha, \beta}\right|^{2} \omega^{2}$ vs $\bar{E}$ for a window $\omega \in(0,0.01)$ in the SIAM. (c) $\left|\rho_{\alpha, \beta}\right|^{2} \omega^{2}$ vs $\omega$ for a window $\bar{E} \in(-0.01,0.01)$ in our model. (d) $\left|\rho_{\alpha, \beta}\right|^{2} \omega^{2}$ vs $\bar{E}$ for a window $\omega \in(0,0.01)$ in our model. The voltage bias is set to $V=1$. The other parameters are as same as those in Fig. 2

ments of density matrix. To be specific, we plot $\left|\rho_{\alpha, \beta}\right|^{2} \omega^{2}$ vs $\omega$ for a small window $\bar{E} \in(-0.01,0.01)$, and also $\left|\rho_{\alpha, \beta}\right|^{2} \omega^{2}$ vs $\bar{E}$ for a small window $\omega \in(0,0.01)$. Again, the density matrix of SIAM is plotted as a comparison. Fig. 4 makes it clear that the distribution of density matrix has similar feature as the current matrix. And they are qualitatively different in SIAM and our model. We find large outliers in the density-matrix elements of SIAM. Indeed, $\left|\rho_{\alpha, \beta}\right|^{2}$ of SIAM are separated into two classes. A small fraction of $\left|\rho_{\alpha, \beta}\right|^{2}$ is much larger than the others. Conversely, $\left|\rho_{\alpha, \beta}\right|^{2}$ of our model are concentrated around a smooth curve. Fig. 4 makes it clear that the density matrix and current matrix in our model can be expressed in a similar form. Note that Eq. (3) and Eq. (5) are similar to each other. In our model, $I_{\alpha, \beta}$ and $\rho_{\alpha, \beta}$ can be expressed as Eq. (3) and (5), respectively. But in SIAM, neither $I_{\alpha, \beta}$ nor $\rho_{\alpha, \beta}$ can be expressed in such a form. Indeed, ETH and NESSH usually stand simultaneously in a model, or break down simultaneously. Their connection has been discussed in Ref. 24]

Fig. 4 presents evidence not only for the first assumption of NESSH (Eq. (5)) but also for the second assumption, i.e. $\rho_{\alpha, \beta}$ after coarse graining diverges as $1 / \omega$ as $\omega \rightarrow 0$. In the plot of $\left|\rho_{\alpha, \beta}\right|^{2} \omega^{2}$ vs $\omega$, a plateau is clearly seen for $\omega \in(0,2)$, indicating the $1 / \omega$-divergence of $\rho_{\alpha, \beta}$. According to Eq. (5), the function $|f(\bar{E}, \omega)|^{2}$ can be obtained by averaging $\left|\rho_{\alpha, \beta}\right|^{2}$ over a small energy box. By using Eq. (9), we obtain

$$
\rho^{2}(\bar{E}, \omega)=D^{3}(\bar{E}) \omega^{2} \overline{\left|\rho_{\alpha \beta}\right|^{2}},
$$

where $\overline{\left|\rho_{\alpha \beta}\right|^{2}}$ is the average over a square box centered at $(\bar{E}, \omega)$ with the edge 0.2 . $\rho^{2}$ is computed in a similar way as $\left|f_{I}\right|^{2}$. Here the sign of $\rho$ can also be chosen freely, and 
$\rho$ is an even function of $\omega$, being continuous at $\omega=0$.

Fig. 3 the bottom panel plots $\rho$ as a function of $\omega$ with $\bar{E}=0$ fixed. $\rho(\bar{E}, \omega)$ displays a peak structure. But it changes continuously in the vicinity of $\omega=0$. Therefore, it is not difficult to determine the value of $\rho(\bar{E}, 0)$. In Fig. 3, we also see that the characteristic functions $f_{I}$ and $\rho$ change with the system's size. As $N_{0}$ increases, the absolute value of $f_{I}$ decreases, while that of $\rho$ increases. It is worth emphasizing that the expectation of current must be size-independent for large enough $N_{0}$, because it has a well-defined thermodynamic limit. But $f_{I}$ and $\rho$ need not be size-independent. Indeed, our numerics show that $f_{I}$ or $\rho$ do not have thermodynamic limit. It is not a surprise, since the density of states is also divergent as $N_{0} \rightarrow \infty$. There is no paradox here, because the current comes from the product of $f_{I}$ and $\rho$.
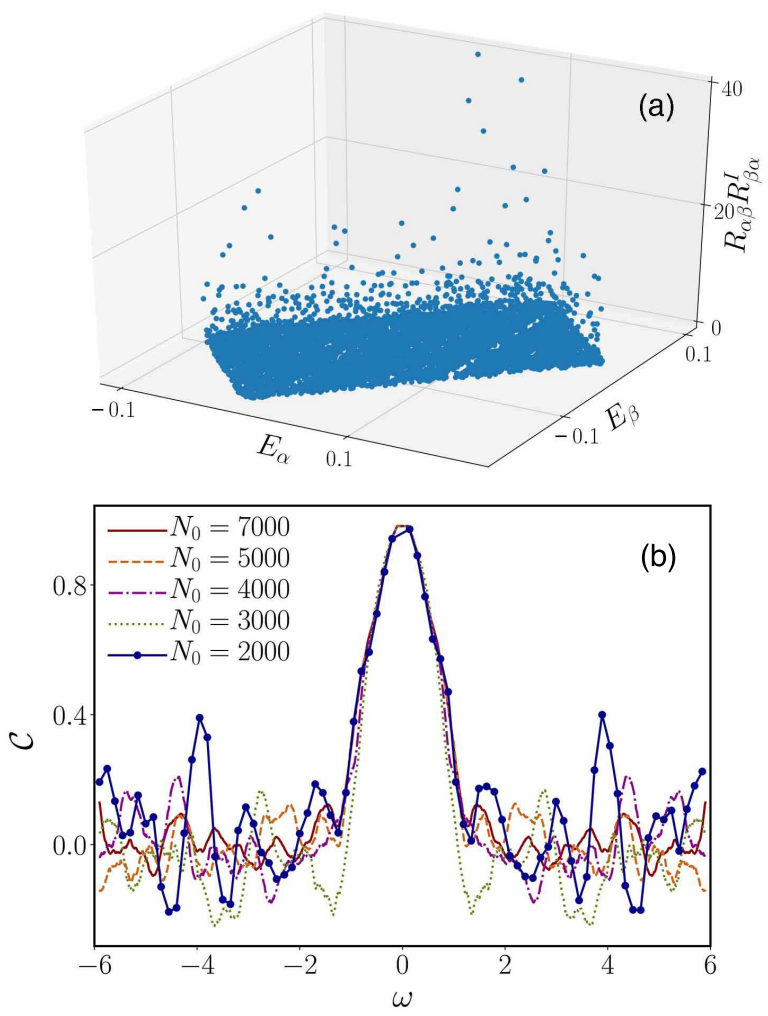

FIG. 5. (Color online) (a) $R_{\alpha \beta} R_{\beta \alpha}^{I}$ vs $(\bar{E}, \omega)$ for the window $\bar{E} \in(-0.1,0.1)$ and $\omega \in(0,0.2)$. (b) $\mathcal{C}$ as a function of $\omega$ for different $N_{0}$. The parameters are as same as those in Fig. 4 .

Finally, we discuss the random number $R_{\alpha \beta} R_{\beta \alpha}^{I}$. Here $R_{\alpha \beta}$ and $R_{\beta \alpha}^{I}$ are real random numbers of zero mean and unit variance, which reflect the fluctuation of $I_{\alpha, \beta}$ and $\rho_{\alpha, \beta}$, respectively. Fig. 5 the top panel plots $R_{\alpha \beta} R_{\beta \alpha}^{I}$ vs $\bar{E}$ and $\omega$ within a small energy box. We see that $R_{\alpha \beta} R_{\beta \alpha}^{I}$ distributes diversely. Due to the correlation between $R_{\alpha \beta}^{I}$ and $R_{\alpha \beta}$, the average of $R_{\alpha \beta} R_{\beta \alpha}^{I}$ is nonzero, which is denoted by $\mathcal{C}$ in above. In practice, $\mathcal{C}$ can be calculated as

$$
\mathcal{C}(\bar{E}, \omega)=\overline{R_{\alpha \beta} R_{\beta \alpha}^{I}},
$$

where the average is over an energy box centered at $(\bar{E}, \omega)$ with the edge chosen to 0.2 . The hermitianity of density matrix and current matrix requires $R_{\alpha \beta}=R_{\beta \alpha}$ and $R_{\alpha \beta}^{I}=R_{\beta \alpha}^{I}$. Therefore, $\mathcal{C}(\bar{E}, \omega)$ is an even function of $\omega$. Fig. 5 the bottom panel plots $\mathcal{C}$ as a function of $\omega$ with $\bar{E}=0$ fixed. It is clear that $\mathcal{C}$ changes smoothly as $\omega$ is in the range $(-1,1)$. And for $\omega \in(-1,1), \mathcal{C}$ is almost independent of $N_{0}$. At least in the vicinity of $\omega=0, \mathcal{C}$ is a well-defined smooth function of $\omega$, indicating that a numerical approach to $\mathcal{C}(\bar{E}, 0)$ is reliable. Since the stationary current depends only upon the value of $\mathcal{C}$ at $\omega=0$, the assumption that $\mathcal{C}$ is a smooth function in deriving Eq. (10) is then reliable.

So far as we can say, our numerics strongly support the assumptions in the derivation of Eq. (10). We then expect Eq. (10) to predict the correct value of current.

\section{CURRENT}

In this section, we discuss the NESS current. There are two different approaches to the stationary current. One is the statistical approach by using the formula (10). The other is the ab-initio approach with respect to definition.

Let us start from the ab-initio approach. According to the definition of NESS current, it is obtained by taking the limit $t \rightarrow \infty$ after $N_{0} \rightarrow \infty$ of Eq. (17). While Eq. (17) already tells us how to calculate the current at arbitrary system's size and arbitrary time by using the eigenenergies and eigenvectors of the Hamiltonian. We use this approach to calculate $I(t)$. Fig. 6 plots the realtime dynamics of current for different $N_{0}$. The bottom panel displays $I(t)$ at exponentially large time scales. It is clear that $I(t)$ for arbitrary $N_{0}$ always decays to zero as $t \rightarrow \infty$. This is what we expect, because the initial imbalance between two leads will be eliminated at a finite period, and the stationary current never survives in a finite system. However, the current will first increase to a finite value and stay there for a while before it decays. Even for $N_{0}=2000$ and $N_{0}=4000$, we already observe a clear plateau of $I(t)$ during the period $10<t<100$. In the top panel of Fig. 6] we see that the plateau is flattened and its lifetime is enhanced as $N_{0}$ increases from 2000 to 7000 . Indeed, as $N_{0}=7000$, the drop of $I$ during the period $10<t<100$ is already insignificant. It is reasonable to conclude that the current will not drop at all as $N_{0}$ goes to infinity. And the height of this plateau must be the NESS current in thermodynamic limit, that is

$$
I=\lim _{t \rightarrow \infty} \lim _{N_{0} \rightarrow \infty} I(t) .
$$

A comparison between the ab-initio result (21) and the statistical formula (10) is done. In previous section, 

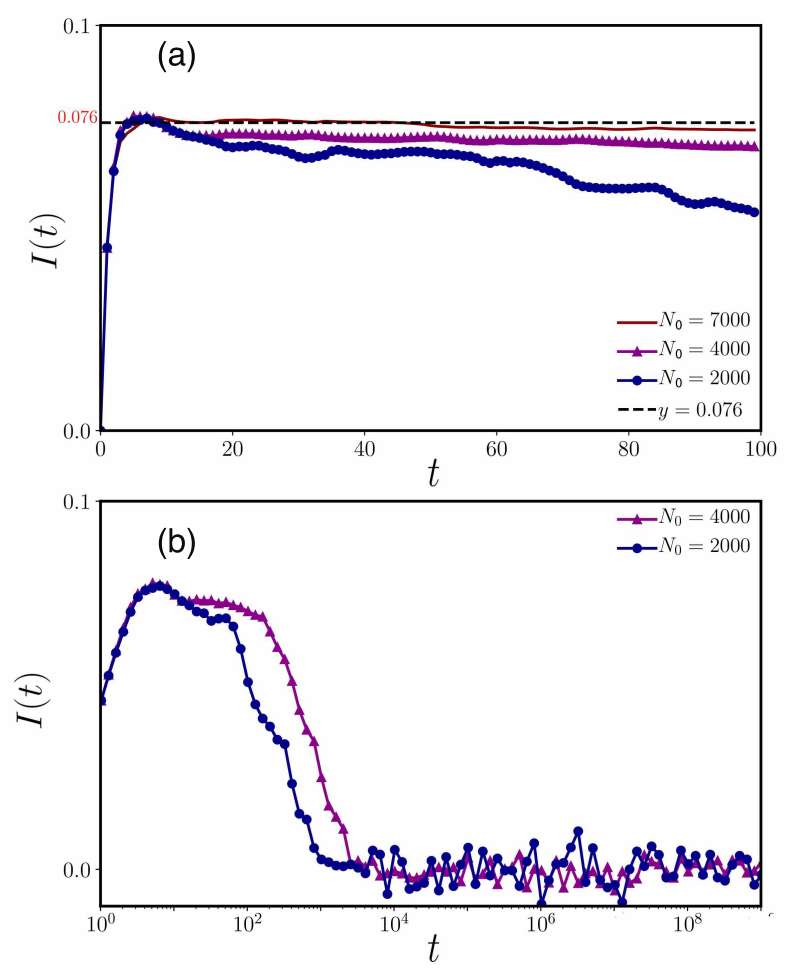

FIG. 6. (Color online) The real-time dynamics of current for different sizes of leads. (a) $I(t)$ for $t \in(0,100)$. (b) $I(t)$ at exponentailly large time scales. We set $\Delta=3, \epsilon_{d}=0$, $N_{0} \sigma=200, V=0.5$ and $\sqrt{D_{l}} \sigma_{t}=1 / \sqrt{10}$ (corresponding to $\Gamma \sim 0.2 \pi$ ). The dashed line in panel (a) is $I=0.076$, which is obtained by the statistical approach.

we already explain how to obtain the characteristic functions: $\rho(\bar{E}, 0), f_{I}\left(\bar{E}, 0^{+}\right)$and $\mathcal{C}(\bar{E}, 0)$. The integral in Eq. (10) is then carried out in a straight forward way. The result of Eq. (10) is found to be $I=0.076$ for the parameters of Fig. 6] which is marked as the dashed line in Fig. 6 the top panel. We see that the plateau of $I(t)$ gradually approaches 0.076 as $N_{0}$ increases. As $N_{0}=7000$, the height of plateau is approximately equal to 0.076 . The statistical formula predicts the stationary current to a high precision, as we expect.

Fig. 7 plots the I-V curve. The solid line comes from the ab-initio calculation, i.e. Eq. (21), while the red dots are the results of the statistical formula (10). Again, the statistical formula and the ab-initio approach predict the same stationary currents for various voltage bias. Our results verify the current formula (10). It is the first time that the statistical formula of stationary current based on NESSH is verified in a specific model. The current is a monotonic function of voltage bias. At small bias, $I$ increases linearly with $V$. But the current saturates at high bias. The shape of $\mathrm{I}-\mathrm{V}$ curve is reminiscent of the inverse of tangent function, while the latter is well known to be the I-V curve of SIAM at zero temperature as the resonant level is located at the center of transport window (see Appendix A for the detail).

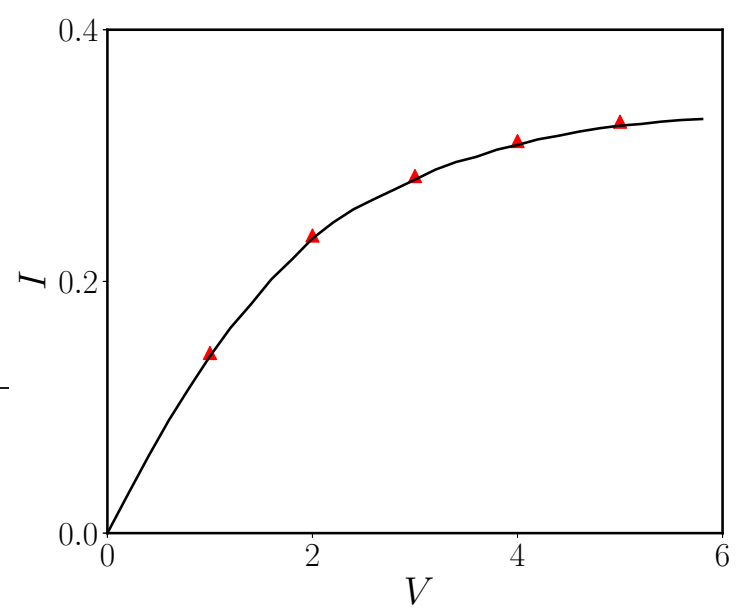

FIG. 7. (Color online) The current as a function of voltage bias. The solid line represents the ab-initio result of stationary current. It is obtained by taking the value of $I(t)$ at $t=40$ for the lead's size being $N_{0}=7000$. The red triangle represents the stationary current obtained by the statistical formula (10). Here we set $\Delta=3, \epsilon_{d}=0, N_{0} \sigma=200, N_{0}=7000$, and $\sqrt{D_{l}} \sigma_{t}=1 / \sqrt{10}$ (corresponding to $\Gamma \sim 0.2 \pi$ ).

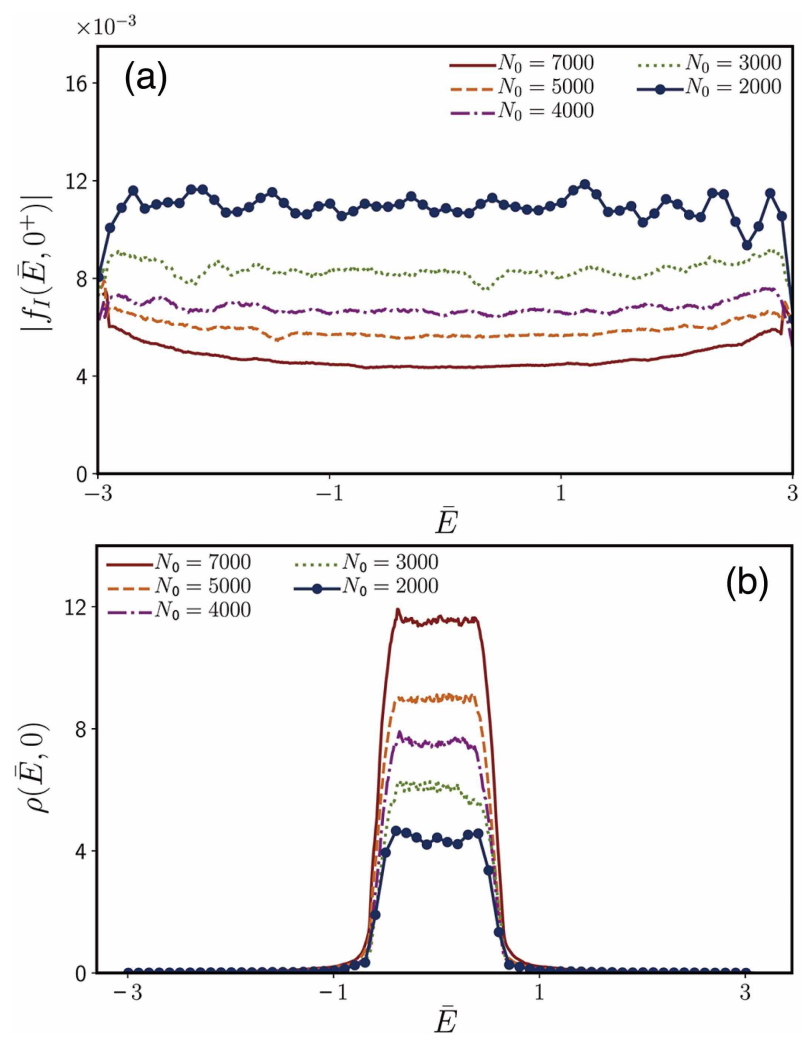

FIG. 8. (Color online) (a) The function $\left|f_{I}\left(\bar{E}, 0^{+}\right)\right|$for different $N_{0}$. (b) The function $\rho(\bar{E}, 0)$ for different $N_{0}$. The voltage bias is set to $V=1$. The other parameters are as same as those in Fig. 1. Especially, $\sqrt{D_{l}} \sigma_{t}=\sqrt{2 / 5}$ corresponds to $\Gamma=0.8 \pi$ 
In the statistical formula, the stationary current is expressed as the integral of the product of $\rho(\bar{E}, 0)$, $f_{I}\left(\bar{E}, 0^{+}\right)$and $\mathcal{C}(\bar{E}, 0)$. To explain the shape of I-V curve, we study these characteristic functions under different voltage bias. Fig. 8(a) displays $\left|f_{I}\right|$ as a function of $\bar{E}$. Note that $f_{I}$ is purely imaginary, therefore, we plot its absolute value. It is clear that $f_{I}$ does not change much with $\bar{E}$. One can approximately take $f_{I}\left(\bar{E}, 0^{+}\right) \approx f_{I}\left(0,0^{+}\right)$as a constant. On the other hand, the function $\rho$ displays a peak structure. In the case of $\epsilon_{d}=0$, the peak is centered at $\bar{E}=0$ (see Fig. $8(\mathrm{~b})$ ). The difference between $\rho$ and $f_{I}$ is that $f_{I}$ comes from the observable operator but $\rho$ is from the density matrix. As a consequence, $f_{I}$ is independent of $V$ or the initial occupation of particles but $\rho$ depends on it.

It is worth mentioning that both $f_{I}$ and $\rho$ depend on the lead's size. The magnitude of $f_{I}$ decreases as $N_{0}$ increases, while that of $\rho$ increases. $f_{I}$ and $\rho$ do not have a well-defined limit as $N_{0} \rightarrow \infty$ (the thermodynamic limit). Only the current has a well-defined thermodynamic limit.

The shape of $\rho(\bar{E}, 0)$ depends both on the initial occupation and the position of the resonant level. Fig. 9(a) displays $\rho(\bar{E}, 0)$ at different voltage bias. Here an important energy scale is the resonant level broadening $\Gamma$, which defines the region of resonant tunneling. When $V$ is less than $\Gamma, \rho$ displays a rectangular peak with a flat top, and the width of the peak is approximately $2 V$. As $V$ increases, the peak becomes wider. But as $V$ goes beyond $\Gamma$, the peak is not rectangular any more. Instead, $\rho$ drops quickly to zero as $|\bar{E}|$ is larger than $\Gamma$. The width of the peak is determined by $\Gamma$, being less than $2 V$. Fig. 9(b) displays $\rho$ at different $\epsilon_{d}$. The center of the peak changes with $\epsilon_{d}$. Indeed, the peak is approximately centered at $\bar{E}=\epsilon_{d}$. But the shape of $\rho$ is indifferent to $\epsilon_{d}$.

The shape of $\rho(\bar{E}, 0)$ is reminiscent of the transmission coefficient. For the SIAM with uniform levelspacing in leads, the transmission coefficient is well known to be approximately $1 /\left(\left(E-\epsilon_{d}\right)^{2}+\Gamma^{2}\right)$ (see Appendix A). We see that $\rho(\bar{E}, 0)$ has a similar shape as $\mathbf{1}_{V} /\left(\left(\bar{E}-\epsilon_{d}\right)^{2}+\Gamma^{2}\right)$, where $\mathbf{1}_{V}$ is the indicator function which equals to 1 in the range $[-V / 2, V / 2]$ but zero otherwise. As is well known, in the transport through a resonant level, the particle can tunnel from one lead to the other if its energy is close to the resonant level, otherwise, the particle is blocked. It is the particles of energy around the resonant level which contribute mainly to the current, while the other particles contribute little. This fact is reflected by the peak structure of $\rho(\bar{E}, 0)$.

Fig. 9 also plots the correlation function $\mathcal{C}(\bar{E}, 0)$ at different $V$ and $\epsilon_{d}$. As similar as $\rho$, the correlation function displays a peak centered at $\epsilon_{d}$, and the width of the peak is approximately equal to that of $\rho$. But the peak of $\mathcal{C}$ is always rectangular with a flat top for large or small $V$. More important, the height of the peak is independent of $V$. Therefore, when calculating the integral of $\rho f_{I} \mathcal{C}$, one can approximately treat $\mathcal{C}$ as a constant, just like $f_{I}$. How the current changes with the voltage bias is mainly determined by the shape of $\rho(\bar{E}, 0)$. Since the function
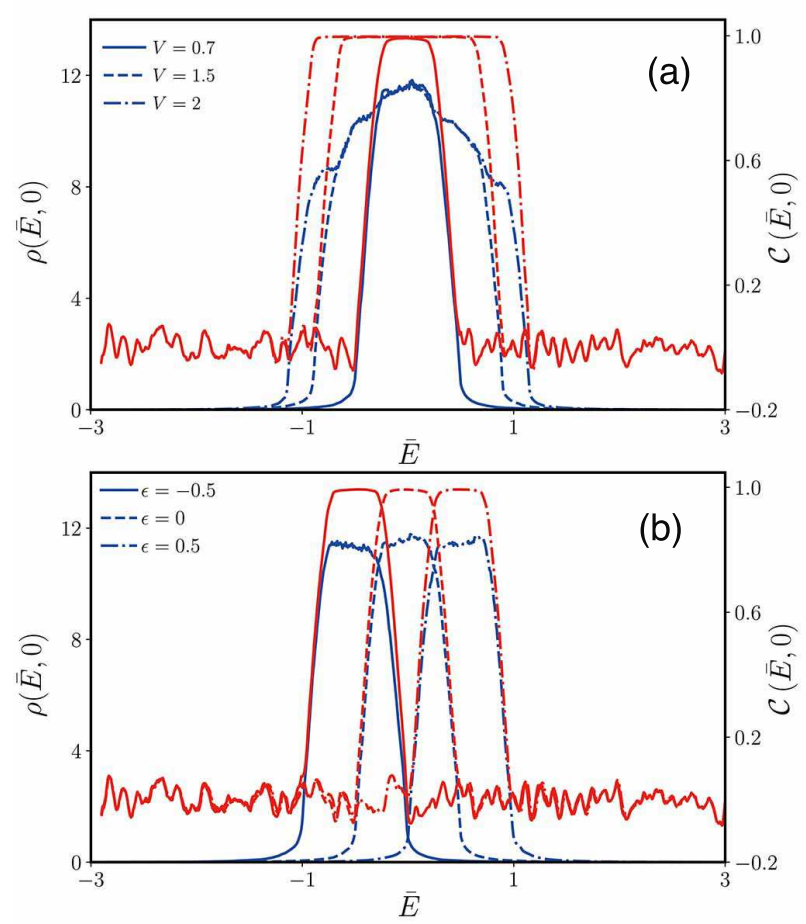

FIG. 9. (Color online) (a) Three bottom lines (blue) plot the function $\rho(\bar{E}, 0)$ at different voltage bias, while three top lines (red) plot $\mathcal{C}(\bar{E}, 0)$. The functions at different $V$ are distinguished by different linetypes. The red and blue lines follow the same legend. In this panel, we set $\Delta=3, \epsilon_{d}=0$, $N_{o} \sigma=200, N_{0}=7000$ and $\sqrt{D_{l}} \sigma_{t}=1 / \sqrt{10}$ (corresponding to $\Gamma \sim 0.2 \pi$ ). (b) We fix $V=0.7$ and plot the functions $\rho$ (three bottom lines) and $\mathcal{C}$ (three top lines) at different $\epsilon_{d}$. $\epsilon_{d}=-0.5,0,0.5$ corresponds to the solid, dashed and dotted lines, respectively.

$\rho(\bar{E}, 0)$ has a similar shape as the transmission coefficient of SIAM, we expect the I-V curve of our model is also similar to that of SIAM. This is what we observe in Fig. 7. If we compare SIAM with our model, it becomes clear that the I-V curve is robust against replacing the uniformly-distributed levels by ones with Wigner-Dyson distribution or replacing the constant coupling by random coupling.

\section{SUMMARY}

In the orthodox approach of studying mesoscopic transport, the initial state is chosen to an equilibrium ensemble with the leads being at different temperatures or chemical potentials. The observables such as current are calculated by using an evolution approach. After infinitely long evolution, the expectation value of observable is believed to be equal to what is measured in experiments, even no experimentalist has ever initialized their laboratory system in such an equilibrium ensemble. NESSH provides a possible explanation for the coinci- 
dence between experimental observation and theoretical method, by stating that the microscopic properties of initial state are unimportant in determining the long-time evolution of observable. Especially, a current formula was derived, which connects the stationary current to a few statistical quantities of initial state, dubbed the characteristic functions.

In this paper, we propose a specific model to check the assumptions of NESSH and the current formula. By modeling the leads as random matrices and the coupling between leads and resonant level as random numbers, we find that the density matrix and current matrix fit well with the description of NESSH and ETH, respectively. We then compute the stationary current by the ab-initio approach and by using the current formula of NESSH. They do agree with each other to a high precision. According to NESSH, the stationary current is an integral of the product of characteristic functions with respect to energy. The characteristic functions $f_{I}, \rho$ and $\mathcal{C}$ are the coarse-grained versions of current matrix, density matrix and their correlation, respectively. $\rho$ as a function of energy displays a peak structure, which has a similar shape as the transmission coefficient of SIAM. As a consequence, the I-V curve of our model is similar to that of SIAM with regular leads and constant coupling.

The initial density matrix and current matrix look like random matrices in the eigenbasis of Hamiltonian. This is the key feature of our model which is distinguished from previous models of mesoscopic transport such as SIAM. In our model, the matrix elements have no large outliers, but in SIAM, a small fraction of elements are dominating the others. The concentrated distribution of off-diagonal elements around a smooth curve is the prerequisite of defining the characteristic functions and applying the current formula of NESSH. In our model, the stationary current is uniquely determined by the values of $\rho, f_{I}$ and $\mathcal{C}$ in the limit $\omega \rightarrow 0$. This is a nonequilibrium version of memory loss. After the system evolves into a NESS, most elements of its initial density matrix have no contribution to physical observables. Only the memory of a few statistical quantities is kept in the off-diagonal elements with infinitesimal energy difference. Our model bridge two different branches of physics - mesoscopic transport and quantum chaos theory, and serves as a benchmark for the future study of transport phenomena by the statistical approach based on NESSH.

\section{ACKNOWLEDGEMENTS}

This work is supported by NSF of China under Grant Nos. 11774315 and 11835011. Pei Wang is also supported by the Junior Associates programm of the Abdus Salam International Center for Theoretical Physics.

\section{Appendix A: Single impurity Anderson model}

The single impurity Anderson model (SIAM) describes a resonant level coupled to two leads. The Hamiltonian of SIAM is expressed as

$$
\hat{H}=\sum_{i k} \epsilon_{i k} \hat{c}_{i k}^{\dagger} \hat{c}_{i k}+\epsilon_{d} \hat{d}^{\dagger} \hat{d}+g \sum_{i k}\left(\hat{c}_{i k}^{\dagger} \hat{d}+\text { h.c. }\right)
$$

where $\hat{c}_{i k}^{\dagger}$ and $\hat{c}_{i k}$ are the fermionic field operators, $i=$ 1,2 denotes the left and right lead, respectively, and $k$ is the index of energy levels in each lead. In each lead, the energy levels have a uniform distribution with the spacing between two neighbors being a constant. We use $N_{A}$ to denote the number of levels in each lead. The level position is then $\epsilon_{i k}=-\Delta+k \frac{2 \Delta}{N_{A}-1}$ with $k=0,1, \cdots, N_{A}-1$, where $2 \Delta$ denotes the bandwidth. Moreover, $\hat{d}$ and $\hat{d}^{\dagger}$ denote the field operators of the resonant level, and $\epsilon_{d}$ denotes its energy. $g$ is the coupling between the resonant level and leads. In this papers, we set $\Delta=3, N_{A}=2000, \epsilon_{d}=0$ and $g \sqrt{N_{A} /(2 \Delta)}=1 / \sqrt{10}$.

The current through the resonant level is usually defined to be

$$
\begin{aligned}
\hat{I} & =\frac{1}{2}\left(\frac{d \hat{N}_{2}}{d t}-\frac{d \hat{N}_{1}}{d t}\right) \\
& =\frac{i}{2} \sum_{k, j=1}^{2}(-1)^{j} g\left(\hat{c}_{j k}^{\dagger} \hat{d}-\hat{d}^{\dagger} \hat{c}_{j k}\right),
\end{aligned}
$$

where $\hat{N}_{j}=\sum_{k} \hat{c}_{j k}^{\dagger} \hat{c}_{j k}$ is the number of particles in lead $j$, and $i$ denotes the imaginary unit. The traditional approach of calculating the stationary current is by using the Keldysh Green's functions ${ }^{30}$. Here an important concept is the level broadening, which comes from the fact that the coupling between resonant level and leads causes uncertainty in the position of resonant level. It is defined to be $\Gamma=2 \pi D_{l} g^{2}$, where $D_{l}=N_{A} /(2 \Delta)$ is the equal density of states in both leads. With our choice of parameters, the level broadening is $\Gamma=0.2 \pi$. The current has a simple expression if the bandwidth $2 \Delta$ is much larger than $\Gamma$. In this case, the transmission coefficient is connected to the imaginary part of retarded Green's function, reading

$$
T(E)=\frac{\Gamma^{2}}{\left(E-\epsilon_{d}\right)^{2}+\Gamma^{2}} .
$$

As the chemical potentials of the left and right leads are $V / 2$ and $-V / 2$, respectively, the stationary current at zero temperature reads

$$
I=\frac{1}{2 \pi} \int_{-V / 2}^{V / 2} d E T(E) .
$$

Here we choose the atomic unit $\hbar=1$. For $\epsilon_{d}=0$, the $\mathrm{I}-\mathrm{V}$ curve is the inverse of tangent function. 
* wangpei@zjnu.cn

E. Wigner, Ann. Math. 62, 548 (1955).

E. Wigner, Ann. Math. 65, 203 (1957).

E. Wigner, Ann. Math. 67, 325 (1958).

${ }^{4}$ F. J. Dyson, J. Math. Phys. 3, 140 (1962).

5 J. M. Deutsch, Phys. Rev. A 43, 2046 (1991).

${ }^{6}$ M. Srednicki, Phys. Rev. E 50, 888 (1994).

7 L. D'Alessio, Y. Kafri, A. Polkovnikov, and M. Rigol, Adv. Phys. 65, 239 (2016)

8 M. Rigol, V. Dunjko, and M. Olshanii, Nature 452, 854 (2008)

9 C. P. Umbach, S. Washburn, R. B. Laibowitz, and R. A. Webb, Phys. Rev. B 30, 4048 (1984).

10 R. A. Webb, S. Washburn, C. P. Umbach, and R. B. Laibowitz, Phys. Rev. Lett. 54, 2696 (1985).

11 M. A. Reed, J. N. Randall, R. J. Aggarwal, R. J. Matyi, T. M. Moore, and A. E. Wetsel, Phys. Rev. Lett. 60, 535 (1988).

12 R. Landauer, IBM J. Res. Dev. 1, 233 (1957).

13 R. Landauer, Philos. Mag. 21, 863 (1970).

14 M. Büttiker, Phys. Rev. Lett. 57, 1761 (1986).

15 Y. Meir and N. S. Wingreen, Phys. Rev. Lett. 68, 2512 (1992).

16 Y. Meir, N. S. Wingreen, and P. A. Lee, Phys. Rev. Lett.
70, 2601 (1993)

17 S. Hershfield, Phys. Rev. Lett. 70, 2134 (1993).

18 P. Bokes and R. W. Godby, Phys. Rev. B 68, 125414 (2003).

19 H. Ness, Phys. Rev. E 88, 022121 (2013).

20 J. A. McLennan, Phys. Rev. 115, 1405 (1959).

21 D. N. Zubarev, Condens. Matter Phys. 4, 7 (1994).

22 In this paper, we do not consider the possibilities of everlasting oscillation in for example a time crystal or nonthermal steady state in an integrable system.

23 P. Wang, J. Stat. Mech.: Theo. Exp. , 093105 (2017)

${ }^{24}$ X. Yang and P. Wang, arXiv:1808.06881 (2018).

${ }^{25}$ C. W. J. Beenakker, Rev. Mod. Phys. 69, 731 (1997)

${ }^{26}$ S. Datta, Electronic Transport in Mesoscopic Systems (Cambridge University Press, Cambridge, 1997).

27 D. I. Pikulin, J. P. Dahlhaus, M. Wimmer, H. Schomerus, and C. W. J. Beenakker, New J. Phys. 14, 125011 (2012).

28 I. Lovas, A. Grabarits, M. Kormos, and G. Zaránd, arXiv:1910.05635 (2019).

29 V. E. Kravtsov, arXiv:0911.0639 (2009).

${ }^{30}$ H. Haug and A.-P. Jauho, Quantum Kinetics in Transport and Optics of Semiconductors (Springer-Verlag, Berlin, Heidelberg 1996). 